\title{
Can Wolbachia be used to control malaria?
}

\author{
Thomas Walker ${ }^{1}$, Luciano Andrade Moreira ${ }^{2}+$ \\ ${ }^{1}$ School of Biological Sciences, The University of Queensland, Brisbane QLD, Australia \\ ${ }^{2}$ Instituto de Pesquisas René Rachou-Fiocruz, Av. Augusto de Lima 1715, 301190-002 Belo Horizonte, MG, Brasil
}

\begin{abstract}
Malaria is a mosquito-borne infectious disease caused by Plasmodium parasites transmitted by the infectious bite of Anopheles mosquitoes. Vector control of malaria has predominantly focused on targeting the adult mosquito through insecticides and bed nets. However, current vector control methods are often not sustainable for long periods so alternative methods are needed. A novel biocontrol approach for mosquito-borne diseases has recently been proposed, it uses maternally inherited endosymbiotic Wolbachia bacteria transinfected into mosquitoes in order to interfere with pathogen transmission. Transinfected Wolbachia strains in Aedes aegypti mosquitoes, the primary vector of dengue fever, directly inhibit pathogen replication, including Plasmodium gallinaceum, and also affect mosquito reproduction to allow Wolbachia to spread through mosquito populations. In addition, transient Wolbachia infections in Anopheles gambiae significantly reduce Plasmodium levels. Here we review the prospects of using a Wolbachiabased approach to reduce human malaria transmission through transinfection of Anopheles mosquitoes.
\end{abstract}

Key words: malaria - Anopheles - Plasmodium - Wolbachia - transinfection

Novel methods of malaria vector control - Malaria is a mosquito-borne infectious disease caused by Plasmodium parasites transmitted by the bite of infected Anopheles mosquitoes. The disease is responsible for approximately 800,000 deaths each year with more than 200 million annual cases (WHO 2010). Vector control for malaria has focused primarily on the adult mosquito through insecticides and bednets. Residual house spraying with chemical insecticides such as DDT has been used but is logistically and economically demanding in many developing countries (Curtis \& Mnzava 2000). When used widely in a community, insecticide treated nets treated with pyrethroid insecticides such as permethrin can provide a safe and simple method of control against night biting Anopheles mosquitoes (Hawley et al. 2003). However, both methods are dependent on a single class of insecticides (pyrethroids) leading to widespread development of insecticide-resistance (WHO 2010). As these current methods of malaria control may not be sustainable for long periods due to the increase in insecticide resistance and environmental concerns, evaluation of novel control strategies need to be undertaken. A novel biocontrol approach has been proposed recently that uses maternally inherited endosymbiotic Wolbachia bacteria transinfected into mosquitoes in order to interfere with pathogen transmission.

Wolbachia's phenotypic effects in insect hosts - Wolbachia are Gram-negative, intracellular, endosymbiotic bacteria that manipulate host reproduction to enhance

Financial support: FNIH/Grand Challenges in Global Health Initiative/Bill and Melinda Gates Foundation/NHMRC (to TW and LAM) LAM is a fellow from CNPq, Brazil.

+ Corresponding author: luciano@cpqrr.fiocruz.br

Received 10 March 2011

Accepted 17 May 2011 their vertical transmission (Sinkins et al. 1997). Wolbachia bacteria were first reported within the reproductive tissues of Culex pipiens mosquitoes by Hertig and Wolbach in 1924 and the species was named Wolbachia pipientis (Werren 1997). Infections are extremely widespread within arthropods (Werren et al. 1995, O'Neill et al. 1997, Jeyaprakash \& Hoy 2000) and Wolbachia are present in numerous mosquito genera including Aedes, Culex, Coquillettidia and Mansonia (Kittayapong et al. 2000, Ricci et al. 2002, Dean \& Dobson 2004, Tsai et al. 2006). Wolbachia are maternally transmitted through the egg cytoplasm and are responsible for several reproductive disorders in their insect hosts such as cytoplasmic incompatibility (CI) (Yen \& Barr 1971), parthenogenesis (Stouthamer et al. 1999), feminization (Rousset et al. 1992) and male killing (Hurst et al. 2000). CI results in the generation of unviable offspring when an uninfected female mates with a Wolbachia-infected male (McGraw et al. 2001). In contrast, Wolbachia-infected females can produce viable progeny when they mate with both infected and uninfected males resulting in a selective reproductive advantage over uninfected females (Hoffmann \& Turelli 1997). This CI phenotype, shown in Figure, is induced by Wolbachia in mosquito species and allows the maternally transmitted Wolbachia to efficiently invade host populations without being infectious or moving horizontally between individuals (Hoffmann \& Turelli 1997).

Although Wolbachia usually form non-virulent associations with their hosts (Sinkins et al. 1997), a virulent strain of Wolbachia, wMelPop, has been described that reduces the adult life span of its natural fruit fly host Drosophila melanogaster (Min \& Benzer 1997). This Wolbachia infection appears to be quiescent in the developing immature stages of the fruit fly but enters into a stage of massive over replication once adult flies emerge. The life-shortening phenotype induced by the $w$ MelPop strain was predicted to have potential use in the control of mosquito-borne diseases. As most pathogens need to develop within the mosquito vector for 8-21 


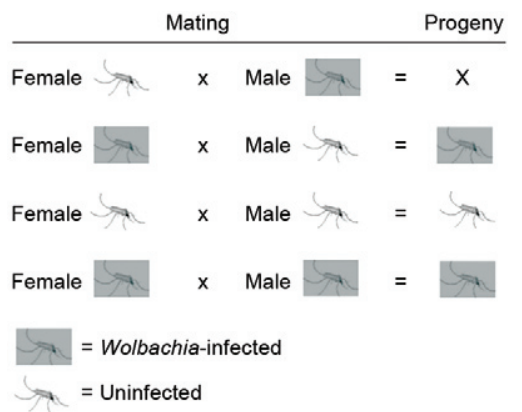

Wolbachia-induced cytoplasmic incompatibility in mosquitoes. The crossing patterns result in unhatched eggs when a Wolbachia-infected male mosquito mates with an uninfected female mosquito. Wolbachia-infected females produce infected progeny in all matings allowing the infection to rapidly spread through mosquito populations.

days post-infection, removal of older mosquitoes from the population, which are responsible for the majority of transmission, may result in substantial reductions in disease transmission (Sinkins \& O'Neill 2000, Brownstein et al. 2003, Rasgon et al. 2003, Cook et al. 2008). However, the major vectors of human diseases such as Anopheles species (malaria) and Aedes aegypti (dengue) do not harbour natural Wolbachia infections. Therefore, the primary aim of using Wolbachia for human disease control is the stable transinfection of the bacteria into medically important mosquito vectors.

Wolbachia transinfection of Ae. aegypti mosquitoes Successful transinfection of Wolbachia between distantly related insect species is dependent on the ability of the $\mathrm{Wol}$ bachia strain to adapt to new intracellular environments (Braig et al. 1994, Xi et al. 2005a). The wAlbB strain of Wolbachia was successfully established in Ae. aegypti using embryo cytoplasm transfer from closely related Aedes albopictus mosquitoes (Xi et al. 2005b). However, to facilitate the transfer of Wolbachia strains from more distantly related insect hosts, mosquito cell line adaptation appears to be critical for transinfection success. In order to facilitate the transfer of $w$ MelPop from D. melanogaster fruit flies into Ae. aegypti mosquitoes, the bacteria was first transferred into mosquito cell lines to allow adaptation to the mosquito intracellular environment (McMeniman et al. 2008). After continuous serial passage in mosquito cell culture for over three years, the mosquito cell lineadapted Wolbachia strain, wMelPop-CLA, was stably introduced into Ae. aegypti using embryo microinjection (McMeniman et al. 2009). Two Wolbachia-infected lines were generated after a period of experimental selection in early generations and both lines remain highly infected four years after establishment.

The $w$ MelPop-CLA strain results in approximately $50 \%$ reduction in the adult lifespan of Ae. aegypti mosquitoes (McMeniman et al. 2009, Yeap et al. 2011). This reduction in adult lifespan of female mosquitoes is predicted to result in a significant reduction in dengue transmission, if the capacity for life-shortening under laboratory conditions can be translated into field settings. Mosquito age is a critical factor for pathogen trans- mission (Dye 1992) as pathogens such as Plasmodium parasites undergoes an extrinsic incubation period (EIP) within the mosquito. The EIP is the time required from the ingestion of the pathogen until it is transmitted to the next vertebrate host. The speed of Plasmodium development within Anopheles mosquitoes depends on host, parasite and environmental factors such as temperature (Paaijmans et al. 2009). A typical incubation period of 10-14 days is relatively long compared to the longevity of adult mosquitoes (Charlwood et al. 1997, Killeen et al. 2000). In addition, there is typically at least two days from adult eclosion until adult female mosquitoes take their first bloodmeal. Adult mosquitoes also experience a high daily mortality rate resulting in only a small percentage of the total population actually surviving long enough to transmit malaria (Brownstein et al. 2003). Therefore, a reduction in the daily survival rates is likely to remove a large proportion of the mosquito population capable of transmitting malaria. Currently the most effective malaria vector control strategies, including indoor residual insecticide sprays and long-lasting insecticidetreated net, reduce the daily survival rates of Anopheles mosquitoes (Enayati \& Hemingway 2010). Reducing the survival of mosquitoes leads to an approximately exponential decline in transmission intensity (Bellan 2010). Consequently, transinfection of life-shortening strains of Wolbachia into Anopheles would be predicted to significantly impact malaria transmission.

Wolbachia-induced pathogen interference - In Drosophila, the wMelPop and closely related Wolbachia strains have the capability of protecting against RNA virus infection by delaying the mortality of flies infected with a range of pathogenic viruses (Hedges et al. 2008, Teixeira et al. 2008). Wolbachia strains also provide significant pathogen protection in mosquitoes (Table). Infection of the $w$ MelPop-CLA strain in Ae. aegypti also provides direct resistance against dengue infection (Moreira et al. 2009). When dengue serotype-2 (DENV-2) was introduced into $w$ MelPop-CLA infected Ae. aegypti mosquitoes by either intrathoracic injection or oral feeding, an almost complete protection against viral infection was observed. The ability of $w$ MelPop-CLA to interfere with viral replication also appears to occur with other arboviruses transmitted by Ae. aegypti, with similar virus interference effects for Chikungunya virus in oral feeding experiments of $w$ MelPop-CLA infected Ae. aegypti (Moreira et al. 2009). There is also further evidence that the wMelPop-CLA strain provides protection against both filarial nematodes (Kambris et al. 2009) and avian malaria parasites (Moreira et al. 2009) suggesting some Wolbachia strains may inhibit a broad range of human pathogens.

As viral interference is not ubiquitous among Wolbachia strains (Moreira et al. 2009, Osborne et al. 2009) the mechanisms behind the ability of Wolbachia to provide resistance against pathogens are unknown. Although immune effector genes are upregulated in wMelPopCLA infected Ae. aegypti mosquitoes, key components of the currently accepted signalling pathways for these effectors do not appear to be transcriptionally modulated by Wolbachia (Moreira et al. 2009, Kambris et al. 2010). Previous studies also revealed that some genes 
TABLE

Wolbachia-induced pathogen protection in mosquitoes

\begin{tabular}{|c|c|c|}
\hline Wolbachia strain & Mosquito species & Pathogen inhibited \\
\hline$w$ MelPop-CLA & Anopheles gambiae ${ }^{a}$ & $\begin{array}{l}\text { Plasmodium berghei } \\
\text { Kambris et al. (2010) } \\
\text { Plasmodium falciparum } \\
\text { Hughes et al. (2011) }\end{array}$ \\
\hline$w$ MelPop-CLA & Aedes aegypti & $\begin{array}{c}\text { Plasmodium gallinaceum } \\
\text { Dengue } \\
\text { Chikungunya } \\
\text { Moreira et al. (2009) } \\
\text { Brugia pahangi } \\
\text { Kambris et al. (2009) }\end{array}$ \\
\hline$w \mathrm{AlbB}$ & Aedes aegypti & $\begin{array}{c}\text { Dengue } \\
\text { Bian et al. (2010) }\end{array}$ \\
\hline$w \mathrm{Mel}$ & Aedes aegypti & $\begin{array}{c}\text { Dengue } \\
\text { T Walker et al., unpublished observations }\end{array}$ \\
\hline$w$ Pip & Culex quinquefasciatus & $\begin{array}{c}\text { West Nile virus } \\
\text { Glaser and Meola (2010) }\end{array}$ \\
\hline
\end{tabular}

$a$ : adult female mosquitoes were transiently infected through intrathoracic injection of Wolbachia. Transinfected (wMelPop-CLA, $w \mathrm{Mel}$ and $w \mathrm{AlbB}$ ) and a natural strain (wPip) of Wolbachia have been shown to directly inhibit pathogens in mosquitoes including two strains of Plasmodium parasites.

from the Toll, Imd and Jak-STAT pathways, implicated in the control of RNA virus infection in insects (Huszar $\&$ Imler 2008) are differentially regulated in Ae. aegypti mosquitoes infected with dengue (Xi et al. 2008). The ability of the $w$ MelPop-CLA strain to provide dengue virus protection may also be dependent on competition for essential host cell components, as DENV-2 infection was only observed in cells of wMelPop-CLA infected mosquitoes that did not harbour Wolbachia (Moreira et al. 2009). Interestingly, cholesterol is a key fatty acid that is obtained from the insect host cell by both Wolbachia (Lin \& Rikihisa 2003, Wu et al. 2004) and arboviruses (Lu et al. 1999, Mackenzie et al. 2007). In addition, Plasmodium is dependent on host lipids in the mosquito stage (Atella et al. 2009), suggesting cholesterol could be a critical host nutrient required by both Wolbachia and mosquito-borne pathogens.

The density and tissue distribution of Wolbachia infections in insect hosts may be an important determinant of their ability to interfere with pathogens. Wolbachia strains that provide protection in Drosophila simulans are closely related to the $w$ MelPop strain in $D$. melanogaster and are found at comparatively high densities in flies (Osborne et al. 2009). The closely-related non-virulent $w \mathrm{Mel}$ strain also provides significant protection against DENV-2 in transinfected Ae. aegypti mosquitoes resulting in complete blockage of dengue transmission under experimental conditions (unpublished observations). Interestingly, strains of Wolbachia that naturally reside in mosquitoes show no or very limited capability for virus protection. Ae. albopictus mosquitoes are infected with non-virulent $w \mathrm{AlbA}$ and $w \mathrm{AlbB}$ strains of Wolbachia (Sinkins et al. 1995 ) yet are competent vectors of dengue virus (Kyle \& Harris 2008). Similarly, Armigeres subaltatus mosquitoes are infected with a Wolbachia strain but no evidence is seen for interference with Japanese encephalitis virus (Tsai et al. 2006). Aedes fluviatillis mosquitoes are infected with a strain of Wolbachia, named wFlu, despite being competent laboratory vectors of Plasmodium gallinaceum (Moreira et al. 2009). Recently the native wPip strain of Wolbachia in Culex quinquefasciatus was shown to have some protective effect against West Nile virus (Glaser \& Meola 2010). However, this effect was much less pronounced when compared to the effects on dengue virus for transinfected fruit fly Wolbachia strains in Ae. aegypti (T Walker et al., unpublished observations). Overall it appears that the ability of Wolbachia to generate pathogen interference is likely to be restricted to Wolbachia strains that grow to high densities and have a wide tissue tropism in their insect host.

Wolbachia transinfection of Anopheles mosquitoes The use of Wolbachia for malaria control will require a stable infection that is transmitted vertically to offspring, as occurs with fruit fly Wolbachia strains transinfected into Ae. aegypti mosquitoes (McMeniman et al. 2009, T Walker et al., unpublished observations). Natural Wolbachia infections had never previously been detected in any species of Anopheles (Curtis \& Sinkins 1998, Kittayapong et al. 2000, Ricci et al. 2002, Tsai et al. 2004, Slotman et al. 2005). However, Wolbachia-positive individuals have recently been found in some species of Anopheles from the Amazon Region (RA Passos \& WP Tadei, personal communication). Further studies on this finding can greatly open the potential use of this methodology for malaria control.

The challenging nature of mosquito microinjection has hampered the progress in transferring Wolbachia between mosquito species. The transfer of Wolbachia 
into mosquito embryos is intrinsically more difficult than transfer into Drosophila, since mosquito embryos are less amenable to inoculation, especially Anopheles embryos. Factors such as the age at the time of injection and desiccation level are especially critical for both injectability and survival of Anopheles embryos. Using Wolbachia-infected mosquito cell lines, the generation of a stably infected line was not been possible despite microinjection of approximately 10,000 embryos of the malaria vectors Anopheles stephensi and Anopheles farauti (unpublished observations).

Wolbachia strains can be maintained in vitro in immunocompetent Anopheles gambiae cell lines (Rasgon et al. 2006, McMeniman et al. 2008), suggesting there is no intrinsic genetic mechanism preventing the infection of Anopheles cells with Wolbachia. In addition, when the $w$ MelPop strain was injected into the hemolymph of $A n$. gambiae adult females, the bacteria were able to survive and replicate in somatic tissues (Jin et al. 2009). However, the infection was not present in germline tissue (ovaries) so a stable transinfection could not be established using this methodology. The reason why Wolbachia does neither naturally infect Anopheles species nor form a stable infection is unknown. The ability to generate transient somatic infections through injection of adult mosquitoes (Jin et al. 2009, Kambris et al. 2010, Hughes et al. 2011) suggests the possibility that Wolbachia may be unable to colonize the germline tissue (ovaries). Successful transinfection requires infection of the germline prior to pole-cell formation in the pre-blastoderm stage of embryo development. Maternal transmission of $\mathrm{Wol}-$ bachia to resulting progeny is dependent on establishing infections in the ovaries of adult females. Ultimately this barrier to germline infection must be overcome to establish stably infected lines that could be deployed for malaria vector control strategies. A recent additional study shows Plasmodium falciparum development in An. gambiae is surpressed by transient somatic infections of $w$ MelPop-CLA (Hughes et al. 2011).

Wolbachia's effect on Plasmodium - Several studies present evidence that Wolbachia is likely to provide some protection against human malaria Plasmodium parasites if stable transinfection of Anopheles is achieved. The effect of the $w$ MelPop-CLA strain on P. gallinaceum was tested as this species of malaria parasite is known to be able to infect $A$ e. aegypti mosquitoes in the laboratory. The $P$. gallinaceum oocyst load was reduced by $67-88 \%$ for $w$ MelPop-CLA infected Ae. aegypti mosquitoes compared to Wolbachia-uninfected mosquitoes seven days after feeding on an infected chicken (Moreira et al. 2009). In An. gambiae females transiently infected with $w$ MelPop using adult injection, mean Plasmodium berghei levels were reduced by $75-84 \%$ (Kambris et al. 2010). Although this combination of vector/parasite does not occur in nature, these results do highlight the ability of Wolbachia to significantly reduce the levels of malaria parasites in Anopheles mosquitoes.

Wolbachia invasion of wild Anopheles mosquito populations - An introduced Wolbachia infection in Anopheles mosquitoes would require induction of the CI phenotype and high rates of maternal transmission to successfully invade wild populations. The $w$ MelPop and $w$ Mel strains induce CI in transinfected Ae. aegypti mosquitoes (McMeniman et al. 2009, T Walker et al., unpublished observations), suggesting this phenotype would be induced in stably infected Anopheles lines. The maternal transmission rate of $w$ MelPop-CLA in Ae. aegypti is estimated to be above $99 \%$ in laboratory lines (McMeniman et al. 2009), suggesting a high infection frequency could be achieved in transinfected Anopheles lines. The potential of Wolbachia to rapidly spread through insect populations under the action of CI was spatially described by Turelli and Hoffmann (1991) in wild D. simulans populations in California with the $w \mathrm{Ri}$ strain rapidly spreading at a rate of $100 \mathrm{~km}$ per year. This rapid spread of Wolbachia through wild Drosophila populations was described as a "Bartonian wave" (Turelli \& Hoffmann 1991). The ability of any Wolbachia strain to successfully invade wild Anopheles mosquito populations will depend on an unstable threshold infection level. This unstable point depends on the negative selection imposed by fitness costs of Wolbachia infection and positive selection associated with CI induction (Hoffmann \& Turelli 1997, Turelli 2010). If the threshold infection frequency is reached through introduction at an initial prevalence greater than the unstable equilibrium value, the Wolbachia infection is expected to spread to fixation over subsequent generations (Hoffmann \& Turelli 1997, Turelli 2010).

Any fitness costs imposed by Wolbachia will raise the unstable point slowing the spread of infection through the "Bartonian wave" of invasion. The likely fitness costs associated with the stable introduction of the wMelPopCLA strain into Anopheles will make this strain relatively weak at spreading into mosquito populations. Alternative Wolbachia strains such as wMel that are predicted to impose less of a fitness cost to transinfected Anopheles mosquitoes are likely to be more successful at invasion of wild mosquito populations. The minimal fitness costs of Wolbachia infection are critical given the importance of mosquito fitness on the ability of released mosquitoes to compete with wild populations. A major advantage of a Wolbachia-based biocontrol approach for malaria is that $\mathrm{CI}$ acts as a self-spreading mechanism for Wolbachia to rapidly invade populations from the release of relatively small numbers of individuals. The predicted direct inhibition of human Plasmodium parasites by Wolbachia may also augment CI as a broad based mechanism for population invasion to provide a positive fitness benefit to Anopheles mosquitoes carrying Wolbachia. This may overlay the traditional "Bartonian" view of CI based invasion dynamics and provide an additional driving force for Wolbachia depending on the extent of fitness advantages conferred to field populations.

Ultimately the use of Wolbachia for malaria control will require stably infected lines of major malaria vectors such as An. gambiae s.l. (Africa), An. stephensi (India) and An. darlingi (Central and South America) and a comprehensive assessment of the protective effect against human malaria parasites such as $P$. falciparum and $P$. vivax. The applied use of Wolbachia for malaria control would also require significant characterization 
of Wolbachia's phenotypic effects in diverse genetic background of these Anopheles vector species. In reality, widespread control of malaria using Wolbachia-based methods is not likely achievable. For example, the difficulties of colonizing An. darlingi (and therefore transinfecting this species with Wolbachia) would prevent the applied use of Wolbachia for control of malaria in parts of the Amazonian Region. In that case, transinfection of colonisable species such as Anopheles aquasalis (Da Silva et al. 2006) would provide applicability in areas where this species has vectorial importance.

Lastly, one has to be aware that the complexity of malaria vector populations (Lanzaro et al. 1998, Donnelly et al. 2002) would be a major complicating factor in the applied use of Wolbachia for malaria control. However, this novel approach may provide an effective mechanism of malaria control in some malaria endemic areas in which a single, vector species is present.

\section{REFERENCES}

Atella GC, Bittencourt-Cunha PR, Nunes RD, Shahabuddin M, SilvaNeto MA 2009. The major insect lipoprotein is a lipid source to mosquito stages of malaria parasite. Acta Trop 109: 159-162.

Bellan SE 2010. The importance of age dependent mortality and the extrinsic incubation period in models of mosquito-borne disease transmission and control. PLOS ONE 5: e10165.

Bian G, Xu Y, Lu P, Xie Y, Xi Z 2010. The endosymbiotic bacterium Wolbachia induces resistance to dengue virus in Aedes aegypti. PLoS Pathog 6: e1000833.

Braig HR, Guzman H, Tesh RB, O’Neill SL 1994. Replacement of the natural Wolbachia symbiont of Drosophila simulans with a mosquito counterpart. Nature 367: 453-455.

Brownstein JS, Hett E, O'Neill SL 2003. The potential of virulent Wolbachia to modulate disease transmission by insects. Jinvertebr Pathol 84: 24-29.

Charlwood JD, Smith T, Billingsley PF, Takken W, Lyimo EOK, Meuwissen JHET 1997. Survival and infection probabilities of anthropophagic anophelines from an area of high prevalence of Plasmodium falciparum in humans. Bull Entomol Res 87: 445-453.

Cook PE, McMeniman CJ, O'Neill SL 2008. Modifying insect population age structure to control vector-borne disease. Adv Exp Med Biol 627: 126-140.

Curtis CF, Mnzava AE 2000. Comparison of house spraying and insecticide-treated nets for malaria control. Bull World Health Organ 78: 1389-1400.

Curtis CF, Sinkins SP 1998. Wolbachia as a possible means of driving genes into populations. Parasitology 116 (Suppl.): S111-S115.

Da Silva AN, Dos Santos CC, Lacerda RN, Santa Rosa EP, De Souza RT, Galiza D, Sucupira I, Conn JE, Póvoa MM 2006. Laboratory colonization of Anopheles aquasalis (Diptera: Culicidae) in Belém, Pará, Brazil. J Med Entomol 43: 107-109.

Dean JL, Dobson SL 2004. Characterization of Wolbachia infections and interspecific crosses of Aedes (Stegomyia) polynesiensis and Ae. (Stegomyia) riversi (Diptera: Culicidae). J Med Entomol 41: 894-900.

Donnelly MJ, Simard F, Lehmann T 2002. Evolutionary studies of malaria vectors. Trends Parasitol 18: 75-80.

Dye C 1992. The analysis of parasite transmission by bloodsucking insects. Annu Rev Entomol 37: 1-19.

Enayati A, Hemingway J 2010. Malaria management: past, present, and future. Annu Rev Entomol 55: 569-591.
Glaser RL, Meola MA 2010. The native Wolbachia endosymbionts of Drosophila melanogaster and Culex quinquefasciatus increase host resistance to West Nile virus infection. PLoS ONE 5: e11977.

Hawley WA, Phillips-Howard PA, ter Kuile FO, Terlouw DJ, Vulule JM, Ombok M, Nahlen BL, Gimnig JE, Kariuki SK, Kolczak MS, Hightower AW 2003. Community-wide effects of permethrintreated bed nets on child mortality and malaria morbidity in western Kenya. Am J Trop Med Hyg 68 (Suppl. 4): 121-127.

Hedges LM, Brownlie JC, O’Neill SL, Johnson KN 2008. Wolbachia and virus protection in insects. Science 322: 702.

Hertig M, Wolbach SB 1924. Studies on the rickettsia-like microorganisms in insects. J Med Res 44: 329-374.

Hoffmann AA, Turelli M 1997. Cytoplasmic incompatibility in insects. In SL O'Neill, AA Hoffmann, JH Werren, Influential passengers: inherited microorganisms and athropod reproduction, Oxford University Press, New York, p. 42-80.

Hughes GL, Koga R, Xue P, Fukatsu T, Rasgon J 2011. Wolbachia infections are virulent and inhibit the human malaria parasite Plasmodium falsiparum in Anopheles gambiae. PLoS Pathog 7: e1002043.

Hurst GD, Johnson AP, Schulenburg JH, Fuyama Y 2000. Male-killing Wolbachia in Drosophila: a temperature-sensitive trait with a threshold bacterial density. Genetics 156: 699-709.

Huszar T, Imler JL 2008. Drosophila viruses and the study of antiviral host-defense. Adv Virus Res 72: 227-265.

Jeyaprakash A, Hoy MA 2000. Long PCR improves Wolbachia DNA amplification: wsp sequences found in $76 \%$ of sixty-three arthropod species. Insect Mol Biol 9: 393-405.

Jin C, Ren X, Rasgon JL 2009. The virulent Wolbachia strain w MelPop efficiently establishes somatic infections in the malaria vector Anopheles gambiae. Appl Environ Microbiol 75: 3373-3376.

Kambris Z, Blagborough AM, Pinto SB, Blagrove MS, Godfray HC, Sinden RE, Sinkins SP 2010. Wolbachia stimulates immune gene expression and inhibits plasmodium development in Anopheles gambiae. PLoS Pathog 6: e1001143.

Kambris Z, Cook PE, Phuc HK, Sinkins SP 2009. Immune activation by life-shortening Wolbachia and reduced filarial competence in mosquitoes. Science 326: 134-136.

Killeen GF, McKenzie FE, Foy BD, Schieffelin C, Billingsley PF, Beier JC 2000. A simplified model for predicting malaria entomologic inoculation rates based on entomologic and parasitologic parameters relevant to control. Am J Trop Med Hyg 62: 535-544.

Kittayapong P, Baisley KJ, Baimai V, O’Neill SL 2000. Distribution and diversity of Wolbachia infections in Southeast Asian mosquitoes (Diptera: Culicidae). J Med Entomol 37: 340-345.

Kyle JL, Harris E 2008. Global spread and persistence of dengue. Annu Rev Microbiol 62: 71-92.

Lanzaro GC, Touré YT, Carnahan J, Zheng L, Dolo G, Traoré S, Petrarca V, Vernick KD, Taylor CE 1998. Complexities in the genetic structure of Anopheles gambiae populations in West Africa as revealed by microsatellite DNA analysis. Proc Natl Acad Sci USA 95: 14260-14265.

Lin M, Rikihisa Y 2003. Ehrlichia chaffeensis and Anaplasma phagocytophilum lack genes for lipid A biosynthesis and incorporate cholesterol for their survival. Infect Immun 71: 5324-5331.

Lu YE, Cassese T, Kielian M 1999. The cholesterol requirement for sindbis virus entry and exit and characterization of a spike protein region involved in cholesterol dependence. J Virol 73: 4272-4278.

Mackenzie JM, Khromykh AA, Parton RG 2007. Cholesterol manipulation by West Nile virus perturbs the cellular immune response. Cell Host Microbe 2: 229-239. 
McGraw EA, Merritt DJ, Droller JN, O’Neill SL 2001. Wolbachiamediated sperm modification is dependent on the host genotype in Drosophila. Proc Biol Sci 268: 2565-2570.

McMeniman CJ, Lane AM, Fong AW, Voronin DA, Iturbe-Ormaetxe I, Yamada R, McGraw EA, O'Neill SL 2008. Host adaptation of a Wolbachia strain after long-term serial passage in mosquito cell lines. Appl Environ Microbiol 74: 6963-6969.

McMeniman CJ, Lane RV, Cass BN, Fong AW, Sidhu M, Wang YF, O'Neill SL 2009. Stable introduction of a life-shortening Wolbachia infection into the mosquito Aedes aegypti. Science 323: 141-144.

Min KT, Benzer S 1997. Wolbachia, normally a symbiont of Drosophila, can be virulent, causing degeneration and early death. Proc Natl Acad Sci USA 94: 10792-10796.

Moreira LA, Iturbe-Ormaetxe I, Jeffery JA, Lu G, Pyke AT, Hedges LM, Rocha BC, Hall-Mendelin S, Day A, Riegler M, Hugo LE, Johnson KN, Kay BH, McGraw EA, van den Hurk AF, Ryan PA, O’Neill SL 2009. A Wolbachia symbiont in Aedes aegypti limits infection with dengue, Chikungunya, and Plasmodium. Cell 139: 1268-1278.

O'Neill SL, Hoffmann AA, Werren JH 1997. Influential passengers: inherited microorganisms and arthropod reproduction, Oxford University Press, Oxford, 214 pp.

Osborne SE, Leong YS, O’Neill SL, Johnson KN 2009. Variation in antiviral protection mediated by different Wolbachia strains in Drosophila simulans. PLoS Pathog 5: e1000656.

Paaijmans KP, Read AF, Thomas MB 2009. Understanding the link between malaria risk and climate. Proc Natl Acad Sci USA 106: 13844-13849.

Rasgon JL, Gamston CE, Ren X 2006. Survival of Wolbachia pipientis in cell-free medium. Appl Environ Microbiol 72: 6934-6937.

Rasgon JL, Styer LM, Scott TW 2003. Wolbachia-induced mortality as a mechanism to modulate pathogen transmission by vector arthropods. J Med Entomol 40: 125-132.

Ricci I, Cancrini G, Gabrielli S, D’Amelio S, Favi G 2002. Searching for Wolbachia (Rickettsiales: Rickettsiaceae) in mosquitoes (Diptera: Culicidae): large polymerase chain reaction survey and new identifications. J Med Entomol 39: 562-567.

Rousset F, Bouchon D, Pintureau B, Juchault P, Solignac M 1992. Wolbachia endosymbionts responsible for various alterations of sexuality in arthropods. Proc Biol Sci 250: 91-98.

Sinkins SP, Braig HR, O’Neill SL 1995. Wolbachia superinfections and the expression of cytoplasmic incompatibility. Proc Biol Sci 261: 325-330.

Sinkins SP, Curtis CF, O’Neill SL 1997. The potential application of inherited symbiont systems to pest control. In SL O'Neill, AA Hoffmann, JH Werren, Influential passengers: inherited microorganisms and athropod reproduction, Oxford University Press, New York, p. 155-175.

Sinkins SP, O'Neill SL 2000. Wolbachia as a vehicle to modify insect populations. In AJA Handler, Insect transgenesis: methods and applications, CRC Press, Boca Raton, p. 271-288.
Slotman M, Della Torre A, Powell JR 2005. Female sterility in hybrids between Anopheles gambiae and A. arabiensis, and the causes of Haldane's rule. Evolution 59: 1016-1026.

Stouthamer R, Breeuwer JA, Hurst GD 1999. Wolbachia pipientis: microbial manipulator of arthropod reproduction. Annu Rev Microbiol 53: 71-102.

Teixeira L, Ferreira A, Ashburner M 2008. The bacterial symbiont Wolbachia induces resistance to RNA viral infections in Drosophila melanogaster. PLoS Biol 6: e2.

Tsai KH, Huang CG, Wu WJ, Chuang CK, Lin CC, Chen WJ 2006. Parallel infection of Japanese encephalitis virus and Wolbachia within cells of mosquito salivary glands. J Med Entomol 43: 752-756.

Tsai KH, Lien JC, Huang CG, Wu WJ, Chen WJ 2004. Molecular (sub) grouping of endosymbiont Wolbachia infection among mosquitoes of Taiwan. J Med Entomol 41: 677-683.

Turelli M 2010. Cytoplasmic incompatibility in overlapping generations. Evolution 64: 232-241.

Turelli M, Hoffmann AA 1991. Rapid spread of an inherited incompatibility factor in California Drosophila. Nature 353: 440-442.

Werren JH 1997. Biology of Wolbachia. Annu Rev Entomol 42: 587-609.

Werren JH, Zhang W, Guo LR 1995. Evolution and phylogeny of Wolbachia: reproductive parasites of arthropods. Proc Biol Sci 261: 55-63.

WHO - World Health Organization 2010. World Malaria Report 2010. Available from: www.who.int/malaria/publications/ atoz/9789241564106/en/index.html.

Wu M, Sun LV, Vamathevan J, Riegler M, Deboy R, Brownlie JC, McGraw EA, Martin W, Esser C, Ahmadinejad N, Wiegand C, Madupu R, Beanan MJ, Brinkac LM, Daugherty SC, Durkin AS, Kolonay JF, Nelson WC, Mohamoud Y, Lee P, Berry K, Young MB, Utterback T, Weidman J, Nierman WC, Paulsen IT, Nelson KE, Tettelin H, O'Neill SL, Eisen JA 2004. Phylogenomics of the reproductive parasite Wolbachia pipientis w Mel: a streamlined genome overrun by mobile genetic elements. PLoS Biol 2: E69.

Xi Z, Dean JL, Khoo C, Dobson SL 2005a. Generation of a novel Wolbachia infection in Aedes albopictus (Asian tiger mosquito) via embryonic microinjection. Insect Biochem Mol Biol 35: 903-910.

Xi Z, Khoo CC, Dobson SL 2005b. Wolbachia establishment and invasion in an Aedes aegypti laboratory population. Science 310: 326-328.

Xi Z, Ramirez JL, Dimopoulos G 2008. The Aedes aegypti toll pathway controls dengue virus infection. PLoS Pathog 4: e1000098.

Yeap HL, Mee P, Walker T, Weeks AR, O’Neill SL, Johnson P, Ritchie SA, Richardson KM, Doig C, Endersby NM, Hoffmann AA 2011. Dynamics of the "popcorn" Wolbachia infection in outbred Aedes aegypti informs prospects for mosquito vector control. Genetics 187: 583-595.

Yen JH, Barr AR 1971. New hypothesis of the cause of cytoplasmic incompatibility in Culex pipiens L. Nature 232: 657-658. 